\title{
Effect of Spinal Alignment Changes on Low Back Pain in Patients Treated with Total Hip Arthroplasty for Hip Osteoarthritis
}

\section{Fumiko Saiki}

The University of Tokyo

\section{Takeyuki Tanaka}

The University of Tokyo

Naohiro Tachibana

The University of Tokyo

\section{Hirofumi Oshima}

The University of Tokyo

\section{Taizo Kaneko}

The University of Tokyo

Chiaki Horii

The University of Tokyo

\section{Hideki Nakamoto}

The University of Tokyo

\section{So Kato}

The University of Tokyo

\section{Toru Doi}

The University of Tokyo

Yoshitaka Matsubayashi

The University of Tokyo

\section{Yuki Taniguchi}

The University of Tokyo

\section{Sakae Tanaka}

The University of Tokyo

Yasushi Oshima ( $\nabla$ yoo-tky@umin.ac.jp )

The University of Tokyo https://orcid.org/0000-0003-4696-1846

\section{Research article}

Keywords: low back pain, THA, hip osteoarthritis, malalignment, scoliosis, pelvic tilt, total hip replacement 
Posted Date: August 5th, 2020

DOl: https://doi.org/10.21203/rs.3.rs-46814/v1

License: (c) (i) This work is licensed under a Creative Commons Attribution 4.0 International License. Read Full License 


\section{Abstract}

\section{Background}

Total hip arthroplasty (THA) is an established procedure for patients with osteoarthritis (OA) of the hip joint that effectively relieves pain and restores function. Because contracture of the hip joint as well as preoperative leg length discrepancy is expected to improve by the surgery, it would be reasonable to speculate that spinal sagittal alignment will also change. However, the influence of spinal alignment changes on clinical symptoms, such as low back pain (LBP), remains controversial. In this study, we aimed to evaluate the associations between spinal alignment changes and improvement in preoperative LBP after THA.

\section{Methods}

From November 2015 to January 2017, 104 consecutive patients who underwent THA were prospectively enrolled. Whole spine X-rays were obtained preoperatively and 12 months postoperatively. The patientreported outcomes (PROs) used were the Numerical Rating Scale (NRS) for back pain, EuroQol 5 Dimension, and Short Form-12. The presence of LBP was defined as an NRS of $\geq 4$. Changes in spinal alignment and PROs before and after surgery were evaluated.

\section{Results}

Seventy-four $(71 \%)$ patients were included in the study. The sagittal spinal parameters changed slightly but significantly; pelvic incidence (PI) decreased, pelvic tilt (PT) increased, and sacral slope decreased. Coronal spinal alignment significantly improved after surgery. Twenty-six (37\%) patients had LBP preoperatively. The patients with preoperative LBP had smaller lumbar lordosis (LL), larger PT, and larger PI minus LL than those in the patients without. Fourteen (54\%) of the 26 patients with preoperative LBP showed improvement, but there were no significant differences in the pre- and postoperative radiographic parameters.

\section{Conclusion}

Although preoperative LBP was likely to resolve after THR, there were no significant correlations between alignment changes and LBP improvement. The cause of LBP in patients with hip OA patients might be multifactorial.

\section{Background}

Correlations among the spine, pelvis, and lower extremities to maintain an upright standing position have been is well established. ${ }^{12} 345$ For example, when a person is standing, the pelvis tilts anteriorly and lumbar lordosis increases. Therefore, when the lumbar spine becomes stiff with aging with a consequent decrease in lumbar lordosis, the acetabulum tilts anteriorly and more flexion is required of the hip joint. However, in patients with osteoarthritis $(\mathrm{OA})$ of the hip joint, contracture of the hip joint can cause 
anteversion of the acetabulum, shortening of the affected limb, obliquity of the pelvis, and spinal sagittal and coronal malalignment. ${ }^{6}$

Total hip arthroplasty (THA) is an established procedure for patients with hip OA that effectively relieves pain and restores function. Because contracture of the hip joint is corrected by the surgery, it would be reasonable to speculate that spinal sagittal alignment will also change. ${ }^{7} 8$ In addition, preoperative leg length discrepancy is expected to improve, which may affect the pelvic obliquity and scoliosis. 910 However, the influence of spinal alignment changes on clinical symptoms, such as low back pain (LBP), remains controversial. ${ }^{11} 12$ In this study, we aimed to evaluate the associations between spinal alignment changes and improvement in preoperative LBP after THA.

\section{Methods}

From November 2015 to January 2017, a total of 104 consecutive patients who underwent THA at our university hospital were prospectively enrolled. All the participants provided written informed consent before participating in the study, which was approved by the Institutional Review Board at the authors' institution. All patients had been diagnosed as having severe symptomatic hip joint OA and underwent THA during the enrollment period.

THA surgery was performed by senior hip surgeons at our institution using a posterior approach. All patients received cementless THA and underwent the routine thromboprophylaxis regimen and postoperative rehabilitation program.

All patients underwent assessments of their whole spine standing X-ray radiographs before and 1 year after surgery. Radiographic sagittal parameters included measurements of pelvic incidence (PI), pelvic tilt (PT), sacral slope (SS), lumbar lordosis (LL), and the distance between the $\mathrm{C7}$ plumb line and posterior corner of the sacrum (C7-SVA). Radiographic coronal parameters included measurements of the distance between the $\mathrm{C} 7$ plumb line and the central sacral vertical line (C7PL-CSVL) and the pelvic obliquity angle, which was defined as the angle between the line connecting the bilateral iliac crests and a horizontal line (Figure 1). The first author performed all the radiographic measurement.

Patients were asked to complete the questionnaires before surgery and at 1-year intervals after surgery. The patient-reported outcomes (PROs) used were the Numerical Rating Scale (NRS) for back pain, EuroQol 5 Dimension (EQ-5D), and Short Form-12 (SF-12). An NRS of $\geq 4$ was defined as the presence of LBP, and improvement supported by $\geq 2$ changes was defined as the improvement of LBP. ${ }^{13} \mathrm{We}$ compared radiographic parameters between patients with and without an improvement of LBP after THA.

SPSS v25 (SPSS Software, IBM Corp., Armonk, NY) was used to perform the Wilcoxon signed rank test and Mann-Whitney $U$ test. A $P$ value of $<0.05$ was regarded as indicative of the statistical significance.

\section{Results}


Of the 104 consecutive patients enrolled, 27 patients who had incomplete data on the questionnaire were excluded. Furthermore, 1 patient was lost to follow-up within 12 months after surgery, 1 patient had dislocation of the hip joint, and 1 patient withdrew informed consent. Therefore, 74 (71\%) patients were eligible for the analysis. As for the hip joint on the contralateral side, 28 patients had mild OA, nine patients had severe $\mathrm{OA}$, and 24 patients had undergone THA for the contralateral side. No patient underwent bilateral THA at the same time. The mean age of patients at surgery was 62 years (2883 years), and 63 (85\%) were women. The pre-and postoperative radiographic parameters are summarized in Table 1. The sagittal spinal parameters changed slightly but significantly, PI decreased, PT increased, and SS decreased. The coronal parameters, specifically, C7-CSVL and the pelvic obliquity angle, significantly improved after surgery. In the PROs, the physical component summary (PCS) in the SF-12 and EQ-5D significantly improved postoperatively (Table 1).

Twenty-six (37\%) patients had LBP before surgery, whereas 48 patients did not. Patients with preoperative LBP showed smaller LL, larger PT, and larger PI minus LL than those of the patients without preoperative LBP (Table 2). In the 26 patients with preoperative LBP, the degree of LBP significantly decreased, with an NRS from 6.0 to $4.8(p<0.01)$. Of these patients, $14(54 \%)$ showed improvement supported by $\geq 2$ changes in the NRS; however, there were no significant differences in the pre-and postoperative radiographic parameters (Tables 3 and 4). 
Table 1

Preoperative and postoperative radiographic parameters and patient-reported outcomes

$\begin{array}{ll}\text { Preoperative } & \text { Postperative (12M) P } \\ (n=74) & (n=74)\end{array}$

\section{Radiographic Parameters}

Sagittal parameters

\begin{tabular}{|c|c|c|c|}
\hline C7-SVA & $41.0 \pm 43.1$ & $37.1 \pm 46.5$ & 0.36 \\
\hline LL & $51.9 \pm 14.4$ & $49.8 \pm 16.6$ & 0.08 \\
\hline PI & $55.4 \pm 10.1$ & $53.5 \pm 10.2$ & 0.01 \\
\hline PT & $15.6 \pm 9.8$ & $17.5 \pm 9.9$ & 0.01 \\
\hline SS & $39.8 \pm 8.3$ & $36.1 \pm 9.7$ & $<0.01$ \\
\hline PI minus LL & $3.5 \pm 15.3$ & $3.7 \pm 17.1$ & 0.51 \\
\hline \multicolumn{4}{|l|}{ Coronal Parameters } \\
\hline C7-CSVL & $12.8 \pm 10.6$ & $7.6 \pm 8.5$ & 0.01 \\
\hline Pelvic Obliquity Angle & $2.6 \pm 3.0$ & $1.6 \pm 2.1$ & $<0.01$ \\
\hline \multicolumn{4}{|l|}{ Patient-Reported Outcomes } \\
\hline LBP (NRS) & $2.8 \pm 2.3$ & $2.4 \pm 2.3$ & 0.15 \\
\hline EQ-5D & $0.74 \pm 0.09$ & $0.85 \pm 0.10$ & $<0.01$ \\
\hline SF-12 PCS & $28.5 \pm 13.0$ & $45.7 \pm 12.9$ & $<0.01$ \\
\hline SF-12 MCS & $54.2 \pm 10.9$ & $56.1 \pm 8.6$ & 0.13 \\
\hline \multicolumn{4}{|c|}{ Data are reported as mean $\pm S D$. } \\
\hline \multicolumn{4}{|c|}{$\begin{array}{l}\text { SVA indicates sagittal vertical axis; } L L, \text { lumbar lordosis; } P \text { I, pelvic incidence; } P T \text {, pelvic tilt; SS, sacral } \\
\text { slope; CSVL, central sacral vertical line; } L B P \text {, low back pain; NRS, numerical rating Scale; EQ-5D, } \\
\text { EuroQol } 5 \text { Dimension; SF-12, Short Form-12; PCS, physical component summary; MCS, mental } \\
\text { component summary. }\end{array}$} \\
\hline
\end{tabular}


Table 2

Comparison of preoperative radiographic parameters and patient-reported outcomes between patients with and without preoperative low back pain

\begin{tabular}{|c|c|c|c|}
\hline & $\mathrm{LBP}+(\mathrm{n}=26)$ & LBP-(N=48) & $\mathbf{p}$ \\
\hline \multicolumn{4}{|l|}{ Radiographic Parameters } \\
\hline \multicolumn{4}{|l|}{ Sagittal Parameters } \\
\hline C7-SVA & $54.8 \pm 52.6$ & $33.4 \pm 34.9$ & 0.26 \\
\hline LL & $45.4 \pm 18.2$ & $55.5 \pm 10.4$ & $<0.01$ \\
\hline PI & $57.6 \pm 10.3$ & $54.2 \pm 10.0$ & 0.12 \\
\hline PT & $19.9 \pm 9.6$ & $13.3 \pm 9.3$ & $<0.01$ \\
\hline SS & $37.7 \pm 8.6$ & $41.0 \pm 8.1$ & 0.13 \\
\hline PI minus LL & $12.2 \pm 18.8$ & $-1.3 \pm 12.4$ & $<0.001$ \\
\hline \multicolumn{4}{|l|}{ Coronal Parameters } \\
\hline C7PL-CSVL & $15.3 \pm 11.9$ & $11.4 \pm 9.9$ & 0.23 \\
\hline Pelvic Obliquity Angle & $2.8 \pm 2.8$ & $2.5 \pm 3.1$ & 0.69 \\
\hline \multicolumn{4}{|c|}{ Patient-Reported Outcomes } \\
\hline LBP (NRS) & $6.0 \pm 1.8$ & $1.1 \pm 0.9$ & $<0.001$ \\
\hline EQ-5D & $0.73 \pm 0.10$ & $0.75 \pm 0.08$ & 0.31 \\
\hline SF-12 PCS & $27.3 \pm 12.0$ & $29.2 \pm 13.5$ & 0.55 \\
\hline SF-12 MCS & $52.4 \pm 10.9$ & $55.1 \pm 10.9$ & 0.49 \\
\hline \multicolumn{4}{|c|}{ Data are reported as mean $\pm S D$. } \\
\hline \multicolumn{4}{|c|}{$\begin{array}{l}\text { SVA indicates sagittal vertical axis; LL, lumbar lordosis; PI, pelvic incidence; PT, pelvic tilt; SS, sacral } \\
\text { slope; CSVL, central sacral vertical line; LBP, low back pain; NRS, numerical rating scale; EQ-5D, } \\
\text { EuroQol } 5 \text { Dimension; SF-12, Short Form-12; PCS, physical component summary; MCS, mental } \\
\text { component summary. }\end{array}$} \\
\hline
\end{tabular}


Table 3

Comparison of preoperative radiographic parameters and patient-reported outcomes in patients with preoperative low back pain (improved vs not improved)

\begin{tabular}{|c|c|c|c|}
\hline & Improved & Not improved & $\mathbf{p}$ \\
\hline & $\mathrm{N}=14$ & $\mathrm{~N}=12$ & \\
\hline Radiographic Parameter & & & \\
\hline Sagittal Parameters & & & \\
\hline C7-SVA & $35.5 \pm 54.0$ & $64.0 \pm 52.8$ & 0.38 \\
\hline LL & $44.5 \pm 17.8$ & $46.5 \pm 19.4$ & 0.98 \\
\hline PI & $56.9 \pm 11.7$ & $57.5 \pm 7.4$ & 0.66 \\
\hline PT & $20.7 \pm 11.7$ & $19.0 \pm 6.8$ & 0.54 \\
\hline SS & $36.2 \pm 7.3$ & $38.5 \pm 9.8$ & 0.57 \\
\hline PI minus LL & $12.4 \pm 20.5$ & $11.8 \pm 17.2$ & 0.98 \\
\hline Coronal Parameters & & & \\
\hline C7PL-CSVL & $15.4 \pm 12.8$ & $15.1 \pm 11.2$ & 0.88 \\
\hline Pelvic Obliquity Angle & $3.1 \pm 3.2$ & $2.5 \pm 2.2$ & 0.62 \\
\hline Patient-Reported Outcom & & & \\
\hline LBP (NRS) & $5.6 \pm 1.9$ & $6.3 \pm 1.8$ & $<0.001$ \\
\hline EQ-5D & $0.74 \pm 0.11$ & $0.72 \pm 0.1$ & 0.81 \\
\hline SF-12 PCS & $29.2 \pm 13.5$ & $25.3 \pm 10.2$ & 0.42 \\
\hline SF-12 MCS & $53.6 \pm 12.8$ & $51.0 \pm 8.7$ & 0.54 \\
\hline Data are reported as me & & & \\
\hline $\begin{array}{l}\text { SVA indicates sagittal ve } \\
\text { slope; CSVL, central sacr } \\
\text { EuroQol } 5 \text { Dimension; SF } \\
\text { component summary. }\end{array}$ & $\begin{array}{l}\text { dosis; Pl, pe } \\
\text { oack pain; } N \\
\text { physical co }\end{array}$ & $\begin{array}{l}\text { cidence; } P T \text {, pel } \\
\text { Imerical rating } S \\
\text { ent summary; } M\end{array}$ & $\begin{array}{l}\text { SS, sacral } \\
-5 D \\
\text { ntal }\end{array}$ \\
\hline
\end{tabular}


Table 4

Comparison of postoperative radiographic parameters and patient-reported outcomes in patients with preoperative low back pain (improved vs not improved)

\begin{tabular}{|c|c|c|c|}
\hline & Improved & Not improved & p \\
\hline & $\mathrm{N}=14$ & $\mathrm{~N}=12$ & \\
\hline Radiographic Parameter & & & \\
\hline Sagittal Parameters & & & \\
\hline C7-SVA & $58.3 \pm 57.3$ & $66.9 \pm 49.3$ & 0.57 \\
\hline LL & $41.2 \pm 21.2$ & $41.7 \pm 24.1$ & 0.92 \\
\hline PI & $56.5 \pm 10.0$ & $53.5 \pm 10.1$ & 0.90 \\
\hline PT & $22.1 \pm 10.4$ & $21.5 \pm 7.3$ & 0.86 \\
\hline ss & $34.4 \pm 8.6$ & $32.1 \pm 10.7$ & 0.50 \\
\hline PI minus LL & $14.9 \pm 22.4$ & $13.0 \pm 21.4$ & 0.88 \\
\hline Coronal Parameters & & & \\
\hline C7PL-CSVL & $12.0 \pm 9.8$ & $10.6 \pm 9.9$ & 0.83 \\
\hline Pelvic Obliquity Angle & $0.9 \pm 0.9$ & $2.4 \pm 3.7$ & 0.65 \\
\hline Patient-Reported Outcon & & & \\
\hline LBP (NRS) & $2.4 \pm 2.0$ & $6.8 \pm 2.3$ & $<0.001$ \\
\hline EQ-5D & $0.86 \pm 0.13$ & $0.76 \pm 0.10$ & 0.04 \\
\hline SF-12 PCS & $47.2 \pm 13.3$ & $36.7 \pm 13.2$ & 0.06 \\
\hline SF-12 MCS & $56.6 \pm 9.8$ & $51.7 \pm 7.6$ & 0.10 \\
\hline Data are reported as me & & & \\
\hline $\begin{array}{l}\text { SVA indicates sagittal ve } \\
\text { slope; CSVL, central sacl } \\
\text { EuroQol } 5 \text { Dimension; SF } \\
\text { component summary. }\end{array}$ & $\begin{array}{l}\text { dosis; } P \text { I, p } \\
\text { ack pain; } \Lambda \\
\text { physical cc }\end{array}$ & $\begin{array}{l}\text { cidence; } P T \text {, pelv } \\
\text { Imerical rating sc } \\
\text { ent summary; } M\end{array}$ & $\begin{array}{l}\text { S, sacral } \\
-5 D, \\
\text { ital }\end{array}$ \\
\hline
\end{tabular}

\section{Discussion}

We sought to assess the changes in and associations of spinal alignment with LBP after THA. In general, coronal alignment significantly improved, whereas sagittal parameters slightly changed. About half of the patients with preoperative LBP showed improvement postoperatively; however, we did not find any relationship between improvement in LBP and either sagittal or coronal alignment changes. 
Hip-spine syndrome was originally described by Offierski and MacNab more than 3 decades ago. ${ }^{1}$ The original concept of this syndrome was based on the fact that patients with hip OA experienced pain relief in the back after being treated for hip OA. The researchers showed that flexion contracture of the hip joint led to increased pelvic forward tilt, lumbar lordosis, and LBP as a result. Because contracture and range of motion of the hip joint improve after THA, it is reasonable to speculate that spinal alignment will change after THA. In this study, we showed that spino-pelvic sagittal parameters slightly changed after THA. PI slightly increased, although it is thought to be an individually constant value. ${ }^{14} 15$ This finding was reasonable considering that the center of the hip joint would shift caudally after THA in patients who experienced central migration of the femoral head preoperatively. Nevertheless, PT slightly increased, whereas SS slightly decreased, which reflected reduced anteversion of the pelvis caused by decreased contracture of the hip joint. These changes were consistent with previous findings, although the difference in the angles was small and might not be clinically significant.

The presence of LBP has been reported in patients with hip OA. According to previous reports, 21.2$56.5 \%$ of patients treated with THA had LBP before surgery, ${ }^{12} 16171819$ which was almost the same as reported in our study (36.6\%). We speculate that the relatively wide range of incidence rates reported in the literature was because of differences in the definition of LBP used among the studies Nevertheless, the incidence of LBP in patients with hip OA is considered relatively high. Moreover, many patients have shown pain relief in the lower back after THA, which accounts for $54-100 \%$ in the literature. ${ }^{7} 12161718$

It is reasonable to speculate that changes in the spino-pelvic alignment might reduce the tension in the back muscles and relieve LBP. 202122 However, the precise mechanism remains elusive. To explain why LBP is relieved after THA, 2 reports focused on the spino-pelvic alignment changes before and after surgery. Weng et al. investigated the effect of THA on sagittal spinal alignment in 69 patients treated with THA. ${ }^{12}$ In their study, 39 (56.5\%) patients complained of LBP before surgery, 17 of whom reported complete resolution and 22 of whom reported significant relief. Although the researchers concluded that the improvement in abnormal sagittal spinal-pelvic-leg alignment helped improve preoperative LBP, they did not show any difference in the radiographic parameters between patients with and without preoperative LBP. Eyvazov et al. investigated the effects of THA on spinal sagittal alignment and static balance in 28 patients. ${ }^{11}$ They showed that LBP and the Oswestry Disability Index (ODI) significantly improved after surgery, but they did not find any significant correlations between postoperative changes in spinal sagittal alignment or postural balance and improvement in LBP and ODI scores. Considering the results from these 2 reports, preoperative LBP improved to some extent after THA; however, the involvement of spinal sagittal malalignment with improvement in LBP remained uncertain.

It is well known that sagittal imbalance can cause LBP. 232425 In our study, patients with preoperative LBP tended to show decreased LL and consequently PI minus LL mismatch as compared to those without LBP. However, although $54 \%$ of the patients with preoperative LBP showed improvement after THA, none of the spinal sagittal parameters were significantly correlated. Therefore, we assume that, although preoperative spinal sagittal malalignment might in part have affected the presence of 
preoperative LBP, factors other than changes in the spinal sagittal alignment must have influenced the improvement in such LBP. Our results do not necessarily eliminate the possibility of an effect caused by slight changes in the sagittal alignment because the number of patients with preoperative LBP was relatively small. Tiny changes in the pelvic anteversion could have influenced the muscle tonus around the lumbar spine and pelvis.

Compared with the changes in the spinal sagittal alignment, coronal balance improved noticeably after THA. This was expected because pelvic obliquity can be mostly corrected after THA as a result of improvement in the leg length discrepancy. It is well known that coronal imbalance can also cause LBP. Eguchi et al. reported that reduction in scoliosis was correlated with an improvement in the Roland-Morris Disability Questionnaire (RDQ) scores in 30 patients undergoing THA. ${ }^{9}$ Although we anticipated that the degree of improvement in the coronal balance would affect the LBP relief after THA, this effect was not observed in our study. We speculate that this finding might be because RDQ can be affected by disorders in the hip joint and in the lumber spine.

Another possibility that could explain the LBP relief after THA is the change in the susceptibility to pain.

${ }^{26}$ Patients with hip OA are always bothered by coxalgia, which could lead to hypersensitivity to pain. In this study, the patients whose preoperative LBP did not improve after THA showed worse quality-of-life outcomes in general, specifically on the EQ-5d and PCS. Although the postoperative mental component summary (MCS) of the SF-12 was not significantly different $(p=0.10)$, it was possible that physical and mental disorders related to the hip joint disorders might have affected the degree of LBP after THA in such patients.

This study has several limitations. First, only one investigator measured the radiographic parameters in this study. Because measurement errors can occur in such cases whose hip OA is severe, examinations by 2 or 3 investigators would have increased the accuracy of the results. Second, although we used a body figure to demonstrate to the patients specifically where the low back was located, the patients might have had difficulty in completely distinguishing LBP from coxalgia. Third, we did not consider pain medications, which would have affected the pain status. Further investigation will be necessary to elucidate these problems.

\section{Conclusion}

LPB was likely to resolve after THA in our patients with hip OA. Although the spinal sagittal and coronal alignment certainly changed after surgery, we did not find significant correlations between alignment changes and LBP improvement. We speculate that changes in the pain threshold might have affected the degree of LBP; however, the underlying mechanism remained uncertain. The cause of LBP in patients with hip OA patients considered to be multifactorial.

\section{List Of Abbreviations}


OA, osteoarthritis; THA, total hip arthroplasty; LBP, low back pain; PI, pelvic incidence; PT, pelvic tilt; SS, sacral slope; LL, lumbar lordosis; C7-SVA, the distance between the C7 plumb line and posterior corner of the sacrum; C7PL-CSVL, the distance between the C7 plumb line and the central sacral vertical line; PROs, patient-reported outcomes; NRS, Numerical Rating Scale; EQ-5D, EuroQol 5 Dimension; SF-12, Short Form12; PCS, physical component summary; ODI, Oswestry Disability Index; RDQ, Roland-Morris Disability Questionnaire; MCS, mental component summary

\section{Declarations}

\section{Ethics approval and consent to participate}

The study approval was given by the Institutional Review Board of the Clinical Research Support Center of The University of Tokyo Hospital (IRB approval number 10965-1). Written informed consent was obtained from all participants. All procedures performed in the studies involving human participants were in accordance with the ethical standards of the institutional and/or national research committee and with the 1964 Helsinki Declaration and its later amendments or comparable ethical standards. All the permissions were obtained in order to access the data used in our study.

\section{Consent for Publication}

All the images used in this article were completed following obtaining a written informed consent from each patient.

\section{Availability of data and material}

The datasets supporting the conclusions of this article are included within the article. The raw data underlying the conclusions made in this study can be inquired to the first author.

\section{Competing interests}

The authors declare that they have no competing interests.

\section{Funding}

No funding was received for this study.

\section{Authors' contributions}


FS: Data collection and manuscript writing. TT: Data collection and analysis. NT: Data collection and analysis. HO: Data collection and analysis. TK: Data collection and analysis. $\mathrm{CH}$ : Data analysis. HN: Data collection and analysis. SK: Data collection and analysis. TD: Data collection and analysis. YM: Data collection and analysis. YT: Data collection and analysis. ST: Supervising the manuscript. YO: Protocol/project development and manuscript editing.

All authors read and approved the final manuscript.

\section{Acknowledgements}

Not applicable.

\section{References}

1. Offierski CM, MacNab I: Hip-spine syndrome. Spine (Phila Pa 1976) 8(3):316-321, 1983.

2. Esposito Cl, Miller TT, Kim HJ, Barlow BT, Wright TM, Padgett DE, Jerabek SA, Mayman DJ. Does Degenerative Lumbar Spine Disease Influence Femoroacetabular Flexion in Patients Undergoing Total Hip Arthroplasty? Clin Orthop Relat Res474(8):1788-1797, 2016.

3. Buckland AJ, Vigdorchik J, Schwab FJ, Errico TJ, Lafage R, Ames C, Bess S, Smith J, Mundis GM, Lafage V. Acetabular Anteversion Changes Due to Spinal Deformity Correction: Bridging the Gap Between Hip and Spine Surgeons. J Bone Joint Surg Am 97(23):1913-1920, 2015.

4. Glassman SD, Bridwell K, Dimar JR, Horton W, Berven S, Schwab F: The impact of positive sagittal balance in adult spinal deformity. Spine (Phila Pa 1976) 30(18):2024-2029, 2005.

5. Lafage V, Schwab F, Patel A, Hawkinson N, Farcy JP: Pelvic tilt and truncal inclination: two key radiographic parameters in the setting of adults with spinal deformity. Spine (Phila Pa 1976) 34(17):E599-606, 2009.

6. Weng WJ, Wang WJ, Wu MD, Xu ZH, Xu LL, Qiu Y. Characteristics of sagittal spine-pelvis-leg alignment in patients with severe hip osteoarthritis. Eur Spine J. 2015;24(6):1228-36.

7. Ben-Galim P, Ben-Galim T, Rand N, Haim A, Hipp J, Dekel S, Floman Y: Hip-spine syndrome: the effect of total hip replacement surgery on low back pain in severe osteoarthritis of the hip. Spine (Phila Pa 1976) 32(19):2099-2102, 2007.

8. Radcliff KE, Orozco F, Molby N, Delasotta L, Chen E, Post Z, Ong A. Change in spinal alignment after total hip arthroplasty. Orthop Surg. 2013;5(4):261-5.

9. Eguchi Y, lida S, Suzuki C, Shinada Y, Shoji T, Takahashi K, Ohtori S. Spinopelvic Alignment and Low Back Pain after Total Hip Replacement Arthroplasty in Patients with Severe Hip Osteoarthritis. Asian Spine J. 2018;12(2):325-34.

10. Abe Y, Sato S, Abe S, Masuda T, Yamada K: The impact of the leg-lengthening total hip arthroplasty on the coronal alignment of the spine. Scoliosis10(Suppl 2):S4, 2015. 
11. Eyvazov K, Eyvazov B, Basar S, Nasto LA, Kanatli U. Effects of total hip arthroplasty on spinal sagittal alignment and static balance: a prospective study on 28 patients. Eur Spine. 2016;J25(11):3615-21.

12. Weng W, Wu H, Wu M, Zhu Y, Qiu Y, Wang W. The effect of total hip arthroplasty on sagittal spinalpelvic-leg alignment and low back pain in patients with severe hip osteoarthritis. Eur Spine $\mathrm{J}$. 2016;25(11):3608-14.

13. Swarm RA, Abernethy AP, Anghelescu DL, Benedetti C, Buga S, Cleeland C, Deleon-Casasola OA, Eilers JG, Ferrell B, Green M, et al. Adult cancer pain. J Natl Compr Canc Netw. 2013;11(8):992-1022.

14. Boulay C, Bollini G, Legaye J, Tardieu C, Prat-Pradal D, Chabrol B, Jouve JL, Duval-Beaupere G, Pelissier J. Pelvic incidence: a predictive factor for three-dimensional acetabular orientation-a preliminary study. Anat Res Int. 2014;2014:594650.

15. Legaye J, Duval-Beaupere G, Hecquet J, Marty C. Pelvic incidence: a fundamental pelvic parameter for three-dimensional regulation of spinal sagittal curves. Eur Spine J. 1998;7(2):99-103.

16. Hsieh PH, Chang Y, Chen DW, Lee MS, Shih HN, Ueng SW. Pain distribution and response to total hip arthroplasty: a prospective observational study in 113 patients with end-stage hip disease. $\mathrm{J}$ Orthop Sci. 2012;17(3):213-8.

17. Parvizi J, Pour AE, Hillibrand A, Goldberg G, Sharkey PF, Rothman RH. Back pain and total hip arthroplasty: a prospective natural history study. Clin Orthop Relat Res. 2010;468(5):1325-30.

18. Staibano P, Winemaker M, Petruccelli D, de Beer J. Total joint arthroplasty and preoperative low back pain. J Arthroplasty. 2014;29(5):867-71.

19. Redmond JM, Gupta A, Hammarstedt JE, Stake CE, Domb BG. The hip-spine syndrome: how does back pain impact the indications and outcomes of hip arthroscopy? Arthroscopy 30(7):872-881, 2014.

20. Lazennec JY, Brusson A, Rousseau MA. Hip-spine relations and sagittal balance clinical consequences. Eur Spine J. 2011;20(Suppl 5):686-98.

21. Tanaka S, Matsumoto S, Fujii K, Tamari K, Mitani S, Tsubahara A. Factors related to low back pain in patients with hip osteoarthritis. J Back Musculoskelet Rehabil. 2015;28(2):409-14.

22. Le Huec JC, Saddiki R, Franke J, Rigal J, Aunoble S. Equilibrium of the human body and the gravity line: the basics. Eur Spine J. 2011;20(Suppl 5):558-63.

23. Le Huec JC, Roussouly P. Sagittal spino-pelvic balance is a crucial analysis for normal and degenerative spine. Eur Spine J. 2011;20(Suppl 5):556-7.

24. Chaleat-Valayer E, Mac-Thiong JM, Paquet J, Berthonnaud E, Siani F, Roussouly P. Sagittal spinopelvic alignment in chronic low back pain. Eur Spine J. 2011;20(Suppl 5):634-40.

25. Schwab FJ, Blondel B, Bess S, Hostin R, Shaffrey Cl, Smith JS, Boachie-Adjei O, Burton DC, Akbarnia BA, Mundis GM, et al: Radiographical spinopelvic parameters and disability in the setting of adult spinal deformity: a prospective multicenter analysis. Spine (Phila Pa 1976) 38(13):E803-812, 2013. 
26. Geneen LJ, Moore RA, Clarke C, Martin D, Colvin LA, Smith BH. Physical activity and Exercise for chronic. pain in adults: an overview of Cochrane Reviews. The Cochrane database of systematic reviews1:CD011279, 2017.

\section{Figures}

A

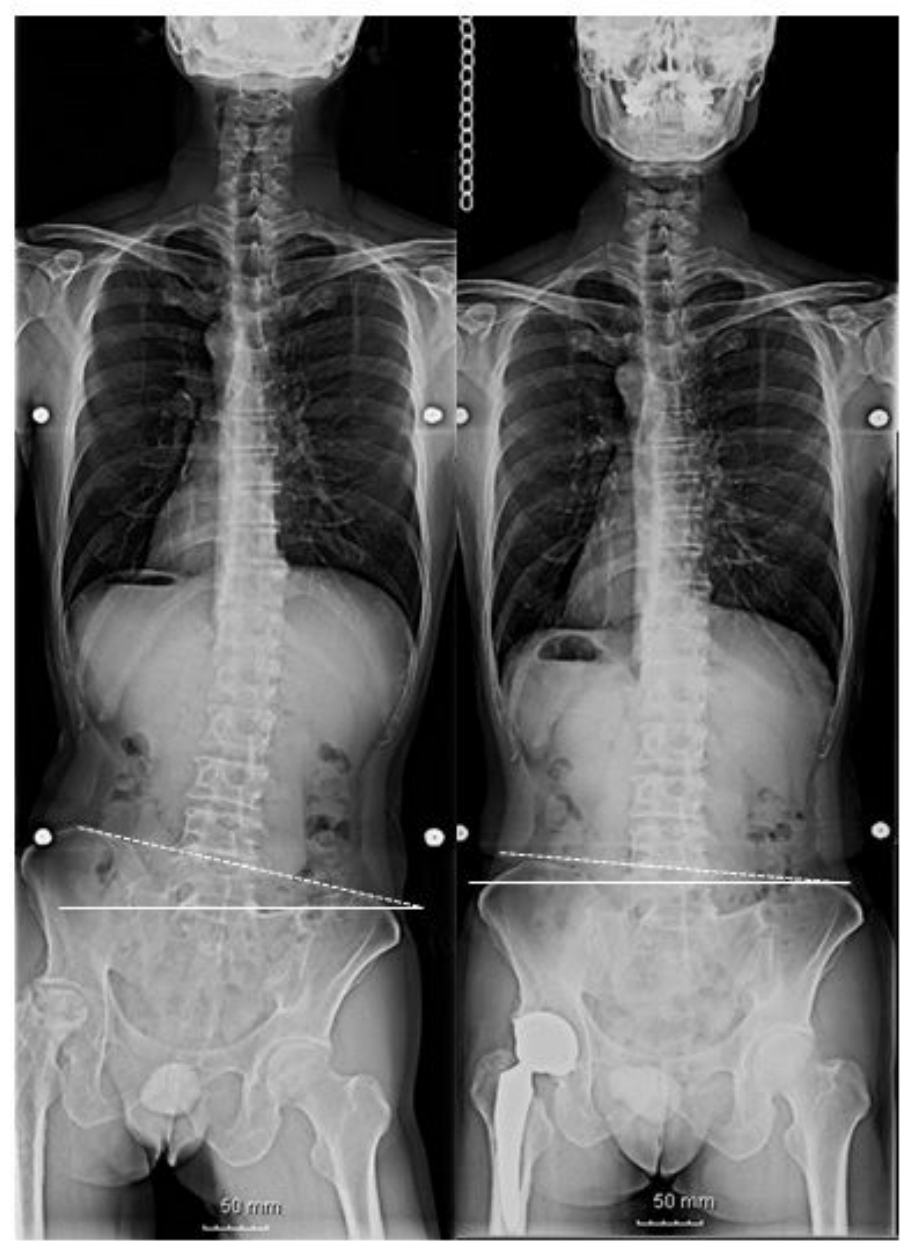

B

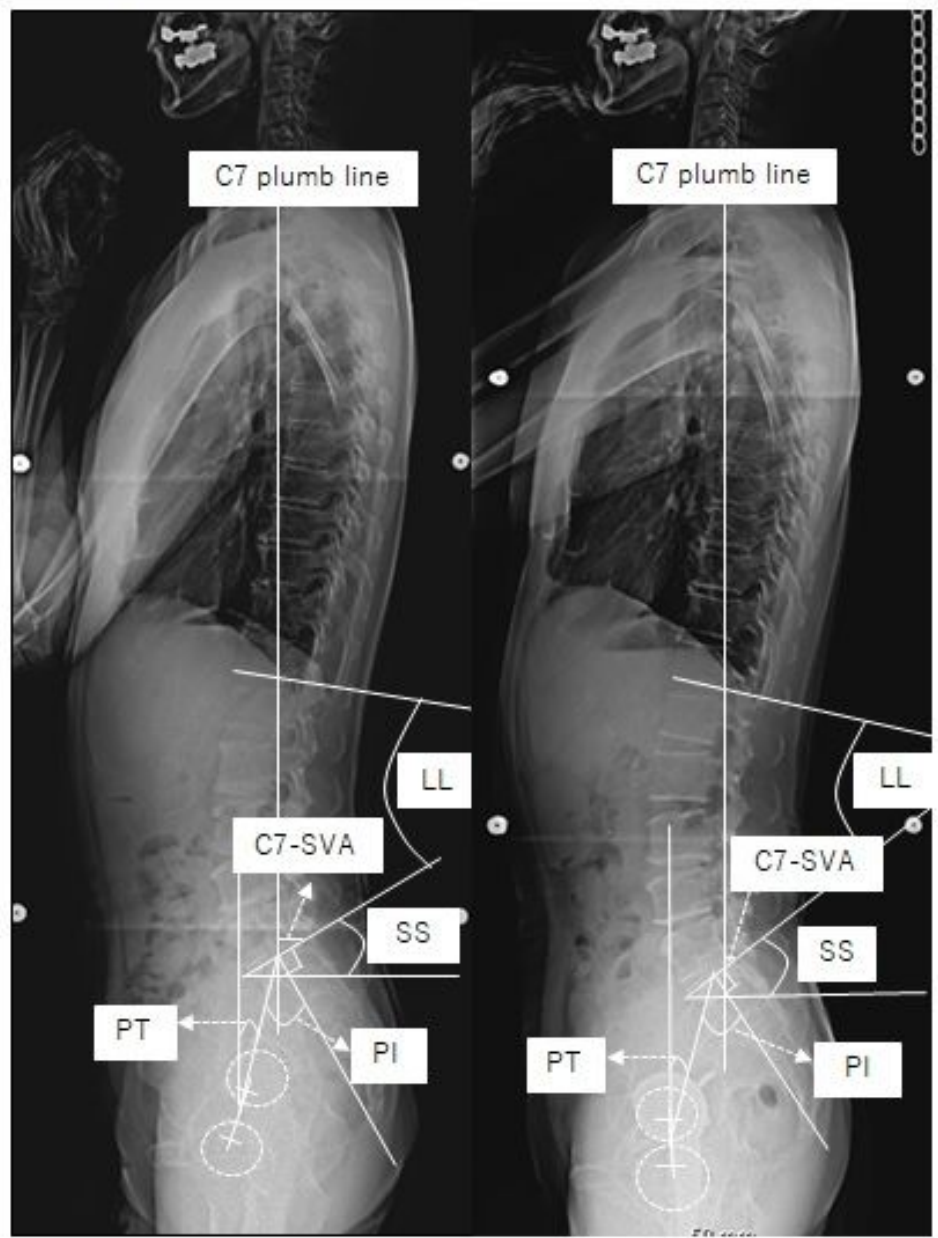

\section{Figure 1}

All patients underwent assessments of their whole spine standing X-ray radiographs before and 1 year after surgery. Radiographic coronal parameters included measurement of the pelvic obliquity angle, which was defined as the angle between the line connecting the bilateral iliac crests (dotted line) and a horizontal line. Radiographic sagittal parameters included measurements of pelvic incidence (PI), pelvic tilt (PT), sacral slope (SS), lumbar lordosis (LL), and the distance between the $\mathrm{C} 7$ plumb line and posterior corner of the sacrum (C7-SVA). 